Algebraic $8 \mathcal{G}$ Geometric $\mathcal{T}_{\text {opology }}$

Volume 4 (2004) 603-622

Published: 20 August 2004

ATG

\title{
Foldable cubical complexes of nonpositive curvature
}

\author{
XiAngDong XIE
}

\begin{abstract}
We study finite foldable cubical complexes of nonpositive curvature (in the sense of A.D. Alexandrov). We show that such a complex $X$ admits a graph of spaces decomposition. It is also shown that when $\operatorname{dim} X=3, X$ contains a closed rank one geodesic in the 1-skeleton unless the universal cover of $X$ is isometric to the product of two CAT(0) cubical complexes.
\end{abstract}

AMS Classification 20F65, 20F67; 53C20

Keywords Rank one geodesic, cubical complex, nonpositive curvature

\section{Introduction}

We study finite foldable cubical complexes with nonpositive curvature (in the sense of A.D. Alexandrov), including the rank rigidity problem of such complexes. Foldable cubical complexes have been studied by W. Ballmann and J. Swiatkowski in $\mathrm{BSw}$. Our notion of foldable cubical complexes is slightly more general than that of $\mathrm{BSw}$. since we do not require gallery connectedness. D. Wise has also studied a class of 2-dimensional cubical complexes $(\mathcal{V H}$ complexes in [W] ) which are closely related to foldable cubical complexes.

A cubical complex $X$ is a CW-complex formed by gluing unit Euclidean cubes together along faces via isometries. We require that all the cubes inject into $X$ and the intersection of the images of two cubes is either empty or equals the image of a cube. The image of a $k$-dimensional unit Euclidean cube in $X$ is called a $k$-cube, and a 1 -cube is also called an edge. Let $d$ be the path pseudometric on $X$. When $X$ is finite dimensional, $d$ is actually a metric and turns $X$ into a complete geodesic space, see $[\mathrm{Br}]$.

Let $X$ be a cubical complex. $X$ is called dimensionally homogeneous if there is an integer $n \geq 1$ such that each cube of $X$ is a face of some $n$-cube. A folding of $X$ is a combinatorial map $f: X \rightarrow C$ onto an $n$-cube $C$ such that the 
restriction of $f$ on each cube is injective. $X$ is foldable if it admits a folding. $X$ has nonpositive curvature in the sense of A.D. Alexandrov if and only if all the vertex links are flag complexes $[\mathrm{BH}]$. Recall that a simplicial complex is a flag complex if any finite set of vertices that is pairwise joined by edges spans a simplex. By a FCC we mean a connected foldable cubical complex that is dimensionally homogeneous, geodesically complete (definition given below) and has nonpositive curvature.

Our first observation about FCCs is the following:

Proposition 2.6 Let $X$ be a FCC of dimension $n$. Then $X$ admits the structure of a graph of spaces, where all the vertex and edge spaces are $(n-1)$ dimensional FCCs and the maps from edge spaces to vertex spaces are combinatorial immersions.

Notice Proposition 2.6 offers the potential for proving statements about FCCs by inducting on dimension.

We shall give two applications of the above observation. The first concerns the rank rigidity problem for $\mathrm{CAT}(0)$ spaces. Let $X$ be a metric space with nonpositive curvature. A curve $\sigma: I \rightarrow X$ is a geodesic if it has constant speed and is locally distance-minimizing. We say $X$ is geodesically complete if every geodesic $\sigma: I \rightarrow X$ can be extended to a geodesic $\tilde{\sigma}: \mathbb{R} \rightarrow X$. A $\mathrm{CAT}(0)$ space is a simply connected complete geodesic space with nonpositive curvature. Let $Y$ be a geodesically complete CAT(0) space. We say $Y$ has higher rank if each geodesic $\sigma: \mathbb{R} \rightarrow Y$ is contained in a flat plane (the image of an isometric embedding $\mathbb{R}^{2} \rightarrow Y$ ). Otherwise we say $Y$ has rank one. There are two main classes of higher rank CAT(0) spaces: symmetric spaces and Euclidean buildings. The following conjecture is still open ([BBu,$[\mathrm{BBr} 2])$ :

Rank Rigidity Conjecture Let $Y$ be a geodesically complete CAT(0) space and $\Gamma$ a group of isometries of $Y$ whose limit set is the entire ideal boundary of $Y$.

(1) If $Y$ has higher rank, then $Y$ isometrically splits unless $Y$ is a symmetric space or an Euclidean building;

(2) If $Y$ has rank one, then $Y$ contains a periodic rank one geodesic.

Recall a complete geodesic $\sigma: \mathbb{R} \rightarrow Y$ is a periodic rank one geodesic if $\sigma$ does not bound a flat half-plane and there is some $\gamma \in \Gamma$ and $c>0$ such that $\gamma(\sigma(t))=\sigma(t+c)$ for all $t \in \mathbb{R}$. The conjecture holds if the action of $\Gamma$ on $Y$ is 
proper and cocompact and $Y$ is a Hadamard manifold $[\mathrm{B}$. or a 2-dimensional polyhedron with a piecewise smooth metric $\mathrm{BBr}$. Claim (1) of the conjecture also holds when $Y$ is a 3 -dimensional piecewise Euclidean polyhedron and $\Gamma$ acts on $Y$ properly and cocompactly $\mathrm{BBr} 2$.

Theorem 3.13 Let $X$ be a finite FCC of dimension 3 with universal cover $\tilde{X}$ and group of deck transformations $\Gamma$.

(1) If $\tilde{X}$ has higher rank, then $\tilde{X}$ is isometric to the product of two $\operatorname{CAT}(0)$ FCCs .

(2) If $\tilde{X}$ has rank one, then $\tilde{X}$ contains a periodic rank one geodesic in the 1 -skeleton.

As the second application, we address the Tits alternative question for finite FCCs . The result has been established by Ballmann and Swiatkowski $[\mathrm{BSw}$. We give a new and very short proof.

Theorem 4.1 Let $X$ be a finite FCC . Then any subgroup of $\pi_{1}(X)$ either contains a free group of rank two or is virtually free abelian.

The paper is organized as follows. In Section 2 we recall some basic facts about FCCs and show that a FCC has a graph of spaces structure. In Section 3 the rank rigidity problem for 3-dimensional FCCs is discussed. In Section 4 we give a new proof of the Tits alternative for FCCs.

The author would like to thank the referee and the editor for many helpful comments.

\section{Foldable cubical complexes}

\subsection{Locally convex subcomplexes}

In this section we show that a FCC has many locally convex subcomplexes. The results in this section are more or less known (see [BSw], C], DJS]). We include the proofs only for completeness.

For any locally finite piecewise Euclidean complex $Y$ and any $y \in Y$, the link $\operatorname{Link}(Y, y)$ is piecewise spherical. We let $d_{y}$ be the induced path metric on $\operatorname{Link}(Y, y)$. If $Y$ has nonpositive curvature, then $\operatorname{Link}(Y, y)$ is a $\operatorname{CAT}(1)$ space. Let $Y$ be a locally finite piecewise Euclidean complex with nonpositive 
curvature and $Z \subset Y$ a subcomplex. For $z \in Z$, a subset $L(Z, z) \subset \operatorname{Link}(Y, z)$ is defined as follows: a point $\xi \in \operatorname{Link}(Y, z)$ belongs to $L(Z, z)$ if there is a nontrivial geodesic segment $z x$ of $Y$ with $z x \subset Z$ such that the initial direction of $z x$ at $z$ is $\xi$.

For any metric space $Z$, the Euclidean cone over $Z$ is the metric space $C(Z)$ defined as follows. As a set $C(Z)=Z \times[0, \infty) / Z \times\{0\}$. The image of $(z, t)$ is denoted by $t z$. $d\left(t_{1} z_{1}, t_{2} z_{2}\right)=t_{1}+t_{2}$ if $d\left(z_{1}, z_{2}\right) \geq \pi$, and $d\left(t_{1} z_{1}, t_{2} z_{2}\right)=$ $\sqrt{t_{1}^{2}+t_{2}^{2}-2 t_{1} t_{2} \cos \left(d\left(z_{1}, z_{2}\right)\right)}$ if $d\left(z_{1}, z_{2}\right) \leq \pi$. The point $O:=Z \times\{0\}$ is called the cone point of $C(Z)$.

Recall a subset $A \subset M$ of a $\mathrm{CAT}(1)$ metric space $M$ is $\pi$-convex if for any $a, b \in A$ with $d(a, b)<\pi$ the geodesic segment $a b$ lies in $A$.

Lemma 2.1 Let $Y$ be a locally finite piecewise Euclidean complex with nonpositive curvature and $Z \subset Y$ a subcomplex. Then $Z$ is locally convex in $Y$ if and only if for each $z \in Z, L(Z, z)$ is $\pi$-convex in $\operatorname{Link}(Y, z)$.

Proof For any $y \in Y$ let $C(\operatorname{Link}(Y, y))$ be the Euclidean cone over $\operatorname{Link}(Y, y)$ and $O$ the cone point. For any $r>0$ let $\bar{B}(y, r) \subset Y$ and $\bar{B}(O, r) \subset$ $C(\operatorname{Link}(Y, y))$ be the closed metric balls with radius $r$. For any subset $A \subset$ $\operatorname{Link}(Y, y)$, let $C_{r}(A) \subset C(\operatorname{Link}(Y, y))$ be the subset consisting of points of the form $t a, t \leq r$ and $a \in A$.

Since $Y$ is a locally finite piecewise Euclidean complex and $Z \subset Y$ is a subcomplex, for each $z \in Z$ there is some $r>0$ and an isometry $g: \bar{B}(z, r) \rightarrow \bar{B}(O, r)$ such that $g(\bar{B}(z, r) \cap Z)=C_{r}(L(Z, z))$. Now it is easy to see that $\bar{B}(z, r) \cap Z$ is convex in $\bar{B}(z, r)$ if and only if $L(Z, z)$ is $\pi$-convex in $\operatorname{Link}(Y, z)$.

Let $X$ be a FCC and $f: X \rightarrow C$ a fixed folding. Two edges $e_{1}$ and $e_{2}$ of $X$ are equivalent if $f\left(e_{1}\right)$ and $f\left(e_{2}\right)$ are parallel in $C$. Let $E_{1}, \cdots, E_{n}$ be the equivalence classes of the edges of $X$. For each nonempty subset $T \subset$ $\{1,2, \cdots, n\}$ we define a subcomplex $X_{T}$ of $X$ as follows: a $k$-cube belongs to $X_{T}$ if all its edges belong to $E_{T}:=\cup_{i \in T} E_{i} . X_{T}$ is disconnected in general. We shall see that each component of $X_{T}$ is locally convex in $X$.

To prove that the components of $X_{T}$ are locally convex in $X$ we also need the following lemma. Recall a spherical simplex is all right if all its edges have length $\pi / 2$, and a piecewise spherical simplicial complex $K$ is all right if all its simplices are all right. When $K$ is finite dimensional, the path pseudometric on $K$ is a metric that makes $K$ a complete geodesic space $[\mathrm{Br}$. 
Lemma 2.2 ( $\mathrm{BH}$, p.211) Let $K$ be a finite dimensional all right spherical complex and $v \in K$ a vertex. If $\sigma:[a, b] \rightarrow K$ is a geodesic such that $\sigma(a), \sigma(b) \notin B(v, \pi / 2)$, then each component of $B(v, \pi / 2) \cap \sigma$ has length $\pi$.

A dimensionally homogeneous cubical complex $Z$ of dimension $n$ has no boundary if each $(n-1)$-cube is contained in the boundaries of at least two $n$-cubes. Similarly, a dimensionally homogeneous simplicial complex $Z$ of dimension $n$ has no boundary if each $(n-1)$-simplex is contained in the boundaries of at least two $n$-simplices.

The following proposition is a consequence of Proposition 1.7.1 of [DJS]. It also follows from Lemmas 1.1 and 1.3 of C]. In addition, W. Ballmann and J. Swiatkowski have made the same observation( $[\mathrm{BSw}$, , Lemma 3.2(4) and the paragraph at the bottom of p.69 and the top of p.70).

Proposition 2.3 Let $X$ be a FCC and $f: X \rightarrow C$ a fixed folding onto an $n$-cube. Then for any nonempty $T \subset\{1,2 \cdots, n\}$, each component of $X_{T}$ is locally convex in $X$. Furthermore, each component of $X_{T}$ is also a FCC .

Proof Let $Z$ be a component of $X_{T}$. For any $z \in Z$ we need to show that $L(Z, z) \subset \operatorname{Link}(X, z)$ is $\pi$-convex. First assume $z$ is a vertex. Then $\operatorname{Link}(X, z)$ is an all right flag complex. By the definition of $X_{T}$ we see $L(Z, z)$ is a full subcomplex of $\operatorname{Link}(X, z)$, that is, a simplex of $\operatorname{Link}(X, z)$ lies in $L(Z, z)$ if and only if all its vertices lie in $L(Z, z)$. Let $\xi, \eta \in L(Z, z)$ with $d_{z}(\xi, \eta)<\pi$. Assume $\xi \eta \nsubseteq L L(Z, z)$. Then there is some $\xi^{\prime} \in \xi \eta-L(Z, z)$. Let $\Delta$ be the smallest simplex of $\operatorname{Link}(X, z)$ containing $\xi^{\prime}$ and $\omega_{1}, \cdots, \omega_{k}$ be its vertices. Then $\xi^{\prime} \in B\left(\omega_{j}, \pi / 2\right)$ for all $1 \leq j \leq k$. Since $L(Z, z)$ is a full subcomplex and $\xi^{\prime} \notin L(Z, z)$, there exists some $j, 1 \leq j \leq k$, with $\omega_{j} \notin L(Z, z)$. We may assume $\omega_{1} \notin L(Z, z)$. By the definition of $X_{T}, \omega_{1}$ corresponds to some edge $e \in E_{i}$ with $i \notin T$. It follows that $\xi, \eta \notin B\left(\omega_{1}, \pi / 2\right)$. Then Lemma 2.2$]$ implies that $\xi \eta \cap B\left(\omega_{1}, \pi / 2\right)$ has length $\pi$, contradicting to the fact that $d_{z}(\xi, \eta)<\pi$.

When $z$ is not a vertex, there is an obvious all right simplicial complex structure on $\operatorname{Link}(X, z)$ where the vertices in $\operatorname{Link}(X, z)$ are represented by geodesic segments parallel to the edges in $X$. In this case $L(Z, z)$ is still a full subcomplex of $\operatorname{Link}(X, z)$ and the above argument applies.

It is clear that $Z$ is a foldable cubical complex that is dimensionally homogeneous. Since $X$ is geodesically complete, it has no boundary. It follows that $Z$ also has no boundary. $Z$ is locally convex in $X$ implies $Z$ has nonpositive curvature in the path metric. By Proposition $2.4 Z$ is geodesically complete and therefore is a FCC. 
Proposition 2.4 Let $Z$ be either a piecewise Euclidean complex that has nonpositive curvature, or a piecewise spherical complex that is locally CAT(1). Assume $Z$ is locally finite and dimensionally homogeneous. Then $Z$ is geodesically complete if and only if it has no boundary.

Proof It is clear that if $Z$ is geodesically complete then it has no boundary. To prove the other direction, we assume $Z$ has no boundary. We first notice that $Z$ is geodesically complete if and only if for any $z \in Z$ and any $\xi \in \operatorname{Link}(Z, z)$ there is some $\eta \in \operatorname{Link}(Z, z)$ with $d_{z}(\xi, \eta) \geq \pi$. The $\operatorname{link} \operatorname{Link}(Z, z)$ is a finite piecewise spherical complex that is CAT(1), dimensionally homogeneous and has no boundary. We prove the following statement by induction on the dimension: Let $Y$ be an $n$-dimensional finite piecewise spherical complex that is CAT(1), dimensionally homogeneous and has no boundary. Then for any $x \in Y$, there is some $y \in Y$ with $d(x, y) \geq \pi$.

When $n=1, Y$ is a finite graph and the claim is clear. Let $n=\operatorname{dim} Y \geq 2$ and suppose there is a point $x \in Y$ such that $d(x, y)<\pi$ for all $y \in Y$. Since $Y$ is finite, there is some $y_{0} \in Y$ with $d(x, y) \leq d\left(x, y_{0}\right)$ for all $y \in Y$. As $Y$ is $\mathrm{CAT}(1)$ and $d\left(x, y_{0}\right)<\pi$, there is a unique minimizing geodesic $\sigma:\left[0, d\left(x, y_{0}\right)\right] \rightarrow Y$ from $y_{0}$ to $x$. Let $\xi \in \operatorname{Link}\left(Y, y_{0}\right)$ be the point represented by $\sigma$. Now $\operatorname{Link}\left(Y, y_{0}\right)$ has dimension $n-1$ and the induction hypothesis implies that there is some $\eta \in \operatorname{Link}\left(Y, y_{0}\right)$ with $d_{y_{0}}(\xi, \eta) \geq \pi$, where $d_{y_{0}}$ is the path metric on $\operatorname{Link}\left(Y, y_{0}\right)$. Hence $\sigma$ can be extended to a geodesic $\tilde{\sigma}$ : $\left[-\epsilon, d\left(x, y_{0}\right)\right] \rightarrow Y$ that contains $y_{0}$ in the interior. As $Y$ is $\operatorname{CAT}(1), \tilde{\sigma}$ is minimizing for small enough $\epsilon$, contradicting to the choice of $y_{0}$.

\subsection{Graph of spaces decomposition}

In this section we show that a FCC admits decomposition as a graph of spaces, as defined in $[\mathrm{SW}]$.

Let $X$ be a FCC and $f: X \rightarrow C_{0}$ a fixed folding onto an $n$-cube. Then the set $E$ of edges of $X$ is a disjoint union $E=\amalg_{i=1}^{n} E_{i}$, see Section 2.1. For each $i$ with $1 \leq i \leq n$, let $T_{i}=\{1,2, \cdots, n\}-\{i\}$. Then the components of $X_{T_{i}}$ are $(n-1)$-dimensional FCCs and are locally convex in $X$.

For each $n$-cube $C$ of $X$, let $e \in E_{i}$ be an edge of $C$ and $C_{i} \subset C$ the hyperplane in $C$ containing the midpoint of $e$ and perpendicular to $e$. It is clear that $C_{i} \subset C$ does not depend on $e$ and is isometric to a $(n-1)$-dimensional unit Euclidean cube. Set $H_{i}=\cup C_{i}$, where $C$ varies over all $n$-cubes of $X . H_{i}$ is not connected in general. An argument similar to the one in Section 2.1] shows 
that each component of $H_{i}$ is locally convex in $X . H_{i}$ has a natural FCC structure, where each $C_{i}$ is a $(n-1)$-cube.

It is not hard to see that each component of $X-X_{T_{i}}$ is isometric to $Y \times(0,1)$, where $Y$ is a component of $H_{i}$. Let $\left\{Y_{1}, \cdots, Y_{k}\right\}$ be the set of components of $H_{i}$. Then $X$ can be obtained from $X_{T_{i}}$ by attaching $Y_{j} \times[0,1]$ along $Y_{j} \times\{0\}$ and $Y_{j} \times\{1\}, 1 \leq j \leq k$. That is, $X$ has the structure of a graph of spaces SW. for each $i, 1 \leq i \leq n=\operatorname{dim} X$. Now we make it more precise. The base graph $G_{i}$ is as follows. The vertex set $\left\{v_{B}\right\}$ of $G_{i}$ is in one-to-one correspondence with the set $\{B\}$ of components of $X_{T_{i}}$, and the edge set $\left\{e_{Y}\right\}$ is in one-toone correspondence with the set $\{Y\}$ of components of $H_{i}$. For each edge $e_{Y}$, consider the component $Y \times(0,1)$ of $X-X_{T_{i}}$ corresponding to $Y$. The closure of $Y \times(0,1)$ in $X$ has nonempty intersection with one or two components of $X_{T_{i}}$. Let $B_{0}, B_{1}$ be these components of $X_{T_{i}}$ (we may have $B_{0}=B_{1}$ ). Then the edge $e_{Y}$ connects the vertices $v_{B_{0}}$ and $v_{B_{1}}$. The vertex space corresponding to $v_{B}$ is the component $B$ of $X_{T_{i}}$ and the edge space corresponding to $e_{Y}$ is the component $Y$ of $H_{i}$.

We notice that the base graph $G_{i}$ is connected: Let $v_{B}, v_{B^{\prime}}$ be two vertices of $G_{i}$. Pick two vertices $v \in B, v^{\prime} \in B^{\prime}$ of $X$. Since $X$ is connected, there are vertices $v_{0}=v, v_{1}, \cdots, v_{l}=v^{\prime}$ such that $v_{j-1}$ and $v_{j}$ are the endpoints of an edge $e_{j}$. Let $B_{j}$ be the component of $X-X_{T_{i}}$ that contains $v_{j}$. If $e_{j} \notin E_{i}$, then $B_{j-1}=B_{j}$. On the other hand, if $e_{j} \in E_{i}$, then $v_{B_{j-1}}$ and $v_{B_{j}}$ are connected by an edge in $G_{i}$.

We next describe the maps from edge spaces to vertex spaces. Let $e_{Y}$ be an edge of $G_{i}$ connecting $v_{B_{0}}$ and $v_{B_{1}}$. We may assume that for each fixed $y \in Y$, $(y, t)$ (where $(y, t) \in Y \times(0,1) \subset X)$ converges to a point in $B_{0}$ as $t \rightarrow 0$ and to a point in $B_{1}$ as $t \rightarrow 1$. The maps $g_{e_{Y}, 0}: Y \rightarrow B_{0}$ and $g_{e_{Y}, 1}: Y \rightarrow B_{1}$ can be described as follows. Recall each $(n-1)$-cube of $Y$ has the form $C_{i}$, where $C$ is an $n$-cube of $X$ and $C_{i} \subset C$ is the hyperplane of $C$ containing the midpoint of some edge $e \in E_{i}$ of $C$ and perpendicular to $e$. Clearly $C$ has the decomposition $C_{i} \times[0,1]$. We may assume $C_{i} \times\{0\} \subset C$ is contained in $B_{0}$ and $C_{i} \times\{1\} \subset C$ is contained in $B_{1}$. Then the map $g_{e_{Y}, 0}: Y \rightarrow B_{0}$ sends $C_{i}$ to $C_{i} \times\{0\}$ via the identity map. Similarly for the map $g_{e_{Y}, 1}$. Thus $g_{e_{Y}, 0}$ and $g_{e_{Y}, 1}$ are nondegenerate combinatorial maps between FCCs. Recall that two cubes in $X$ either are disjoint or intersect in a single cube. It follows that the maps $g_{e_{Y}, 0}$ and $g_{e_{Y}, 1}$ are immersions, that is, they are locally injective.

Lemma 2.5 The maps $g_{e_{Y}, 0}$ and $g_{e_{Y}, 1}$ are local isometric embeddings. In particular, they induce injective homomorphisms between fundamental groups. 
Proof We show that $g_{e_{Y}, 0}$ is a local isometric embedding, the proof for $g_{e_{Y}, 1}$ is similar. It suffices to show that $g_{e_{Y}, 0}: Y \rightarrow B_{0}$ sends geodesics in $Y$ to geodesics in $B_{0}$. Recall that $Y$ is a component of $H_{i}$ and the component of $X-X_{T_{i}}$ containing $Y$ is isometric to $Y \times(0,1) . \quad Y$ can be identified with $Y \times\left\{\frac{1}{2}\right\}$. Let $\sigma: I \rightarrow Y$ be a geodesic. Then for each $t, 0<t<1$, the map $\sigma_{t}: I \rightarrow X$ with $\sigma_{t}(s)=(\sigma(s), t)$ for $s \in I$ is a geodesic in $X$. Since $X$ has nonpositive curvature, the limit map $\sigma_{0}: I \rightarrow X, \sigma_{0}(s):=\lim _{t \rightarrow 0} \sigma_{t}(s)$ is also a geodesic in $X$ (see p.121, Corollary 7.58 of $[\mathrm{BH}]$ ). Now the lemma follows since $\sigma_{0}=g_{e_{Y}, 0} \circ \sigma$.

Summarizing the above observations we have the following:

Proposition 2.6 Let $X$ be a FCC of dimension $n$. Then $X$ admits the structure of a graph of spaces, where all the vertex and edge spaces are $(n-1)$ dimensional FCCs and the maps from edge spaces to vertex spaces are combinatorial immersions. In particular, $\pi_{1}(X)$ has a graph of groups decomposition.

Let $X, G_{i}$ be as above, $v_{B} \in G_{i}$ a vertex and $e_{Y} \subset G_{i}$ an edge incident to $v_{B}$. Suppose for each $y \in Y,(y, t)$ converges to a point in $B$ as $t \rightarrow 0$, and let $g_{e_{Y}, 0}: Y \rightarrow B$ be the map from the edge space to the vertex space. For each vertex $w \in Y, \overline{\{w\} \times(0,1)}$ is an edge in $X$; we let $e_{w}$ be the associated oriented edge which goes from 0 to 1 . For any oriented edge $e$ of $X$ with initial point $v, \vec{e} \in \operatorname{Link}(X, v)$ denotes the point representing $e$.

Lemma 2.7 In the above notation, the following two conditions are equivalent:

(1) $g_{e_{Y}, 0}$ is not a covering map;

(2) there exists a vertex $w \in Y$ and an oriented edge $e \subset B$ with initial point $v:=g_{e_{Y}, 0}(w)$ such that $d_{v}\left(\vec{e}, \overrightarrow{e_{w}}\right) \geq \pi$.

Proof We first notice that (2) is equivalent to the following condition:

(3) There is a vertex $w \in Y$ and an edge $e \subset B$ incident to $v=g_{e_{Y}, 0}(w)$ such that no edge (in $Y$ ) incident to $w$ is mapped to $e$.

So (2) clearly implies (1). Now assume $g_{e_{Y}, 0}$ is not a covering map. If the image of $g_{e_{Y}, 0}$ does not contain all the vertices of $B$, the connectedness of the 1-skeleton of $B$ implies there is an edge $e \subset B$ with two endpoints $v_{1}$ and $v_{2}$ such that $v_{1} \in \operatorname{image}\left(g_{e_{Y}, 0}\right)$ and $v_{2} \notin \operatorname{image}\left(g_{e_{Y}, 0}\right)$. In this case no edge of $Y$ is mapped to $e$ and (3) holds. So we assume image $\left(g_{e_{Y}, 0}\right)$ contains all the vertices of $B$. If (3) does not hold, then image $\left(g_{e_{Y}, 0}\right)=B$ and for each vertex $w \in Y, g_{e_{Y}, 0}$ maps $\operatorname{star}(w)$ isomorphically onto $\operatorname{star}(v)$, where $v=g_{e_{Y}, 0}(w)$. It follows that $g_{e_{Y}, 0}$ is a covering map, contradicting to the assumption. 


\subsection{Davis complexes of right-angled Coxeter groups}

In this section we give examples of finite FCCs whose universal covers are Davis complexes of certain right-angled Coxeter groups.

Let $S$ be a finite set, and $M=\left(m_{s, s^{\prime}}\right)_{s, s^{\prime} \in S}$ a symmetric matrix such that $m_{s, s}=1$ for $s \in S$ and $m_{s, s^{\prime}} \in\{2,3, \cdots,\} \cup\{\infty\}$ for $s \neq s^{\prime} \in S$. The group $W$ given by the presentation $W=<S \mid\left(s s^{\prime}\right)^{m_{s, s^{\prime}}}=1, s, s^{\prime} \in S>$ is a Coxeter group, where $\left(s s^{\prime}\right)^{\infty}=1$ means the relation is void. The Coxeter group $W$ is a right-angled Coxeter group if for any $s \neq s^{\prime} \in S, m_{s, s^{\prime}}=2$ or $\infty$.

Given any Coxeter group $W$, there is a locally finite cell complex $D_{W}$, the so called Davis complex of $W$ such that $W$ acts on $D_{W}$ properly with compact quotient $[\mathrm{D}]$. Moussong ( $[\mathrm{M}], \mathrm{D}])$ showed that there is a piecewise Euclidean metric on $D_{W}$ that turns $D_{W}$ into a CAT(0) space. The action of $W$ on $D_{W}$ preserves the piecewise Euclidean metric, that is, $W$ acts on $D_{W}$ as a group of isometries. When $W$ is a right-angled Coxeter group, the Davis complex $D_{W}$ is a cubical complex. Below we shall describe FCCs which are finite quotients of Davis complexes of certain right-angled Coxeter groups.

There is a one-to-one correspondence between right-angled Coxeter groups and finite flag complexes. Let $W$ be a right-angled Coxeter group with standard generating set $S$. The nerve $N(W)$ of $W$ is a simplicial complex with set of vertices $S$. For any nonempty subset $T \subset S$ there is a simplex in $N(W)$ with $T$ as its vertex set if and only if $m_{t, t^{\prime}}=2$ for any $t \neq t^{\prime} \in T . N(W)$ is clearly a flag complex. Conversely, let $K$ be a finite flag complex with set of vertices $S$. Then we can define a right-angled Coxeter group with generating set $S$ as follows: for $s \neq s^{\prime}, m_{s, s^{\prime}}=2$ if there is an edge in $K$ joining $s$ and $s^{\prime}$, and $m_{s, s^{\prime}}=\infty$ otherwise.

Let $K$ be a finite flag complex with vertex set $S$. We shall construct a finite cubical complex $Y(K)$ whose vertex links are all isomorphic to $K([\mathrm{BH}, \mathrm{p} .212)$. Let $V$ be a Euclidean space with dimension equal to $|S|$, the cardinality of $S$. Identify the standard basis $e_{s}(s \in S)$ with $S$ and consider the unit cube $[0,1]^{|S|} \subset V$. For each nonempty subset $T \subset S$, let $F_{T}$ be the face of $[0,1]^{|S|}$ spanned by the unit vectors $e_{t}, t \in T$. The cubical complex $Y(K)$ is the subcomplex of $[0,1]^{|S|}$ consisting of all faces parallel to $F_{T}$, for all nonempty subsets $T$ of $S$ that are the vertex sets of simplices of $K$. Notice that $Y(K)$ contains the 1 -skeleton of the unit cube $[0,1]^{|S|} \subset V$. In particular, $Y(K)$ is connected. 
Proposition 2.8 $[\mathrm{BH}]$ Let $K$ be a finite flag complex. Then $Y(K)$ is a connected finite cubical complex with nonpositive curvature all of whose vertex links are isomorphic to $K$.

We subdivide $Y(K)$ by using the hyperplanes $x_{s}=1 / 2(s \in S)$ of the unit cube $[0,1]^{|S|}$. Let $X(K)$ be the obtained cubical complex. Then $X(K)$ is also a finite cubical complex of nonpositive curvature with some of its vertex links isomorphic to $K$.

A simplicial complex $K$ is foldable if there is a simplicial map $f: K \rightarrow \Delta$ onto an $n$-simplex such that the restriction of $f$ to each simplex is injective.

Corollary 2.9 Let $K$ be a finite flag complex. If $K$ is foldable, dimensionally homogeneous and has no boundary, then $X(K)$ is a finite FCC .

Proof It is not hard to see that $X(K)$ is dimensionally homogeneous and has no boundary. Proposition 2.4 implies that $X(K)$ is geodesically complete. We need to show that $X(K)$ is foldable. First we notice that the group $\mathbb{Z}_{2}^{|S|}$ acts on $X(K)$ as a group of isometries: the $s$-th factor $\mathbb{Z}_{2}$ acts as the orthogonal reflection about the hyperplane $x_{s}=1 / 2$. Let $o \in X(K)$ be the origin of $V$ and $\operatorname{star}(o)$ the star of $o$ in $X(K)$. Then the quotient of $X(K)$ by $\mathbb{Z}_{2}^{|S|}$ is isomorphic to $\operatorname{star}(o)$. So we have a nondegenerate combinatorial map from $X(K)$ onto star $(o)$. Since the link of $X(K)$ at $o$ is isomorphic to $K$ and $K$ is foldable, the star star $(o)$ can be folded according to the folding of $K$. The composition of these two maps is a folding of $X(K)$.

Next we construct certain flag complexes that satisfy the assumptions in Corollary 2.9. Let $n \geq 2$. A standard $n$-sphere is the unit round $n$-sphere with an all right simplicial complex structure. A standard $n$-sphere has $n+1$ subcomplexes which are standard $(n-1)$-spheres; we call them equators. Let $\mathbb{S}^{n}$ be a standard $n$-sphere and $E$ one of its equators. An all right simplicial complex is called a standard $n$-hemisphere if it is isomorphic to the closure of one of the components of $\mathbb{S}^{n}-E$; the subcomplex of the standard $n$-hemisphere corresponding to $E$ is also called its equator. The unique point on a standard $n$-hemisphere that has distance $\pi / 2$ from its equator is called its pole.

Let $\mathbb{S}^{n}$ be a standard $n$-sphere and $E_{1}, \cdots, E_{n+1}$ its equators. A hemispherex is an all right simplicial complex obtained from $\mathbb{S}^{n}$ by attaching a finite number of standard $n$-hemispheres along the equators of $\mathbb{S}^{n}$ such that for each $i, 1 \leq i \leq n+1$, there is at least one hemisphere attached along $E_{i}$. Here the 
attaching is realized through isomorphisms between equators of $\mathbb{S}^{n}$ and those of the hemispheres. It is clear that a hemispherex $H$ satisfies all the conditions in Corollary [2.9], so the corresponding $X(H)$ is a finite FCC. The universal cover of $X(H)$ is a subdivision of the Davis complex of the right-angled Coxeter group whose nerve is $H$.

Hemispherex was first introduced by Ballmann and Brin in $[\mathrm{BBr} 2$. The Euclidean cone over a hemispherex is the first example of a higher rank CAT(0) space aside from Euclidean buildings and symmetric spaces. But such a space does not admit cocompact isometric actions. On the other hand, we shall see in Section 3.2 that if $X$ is a finite FCC with some vertex link isomorphic to a hemispherex, then it contains closed rank one geodesics. In particular, $X(H)$ contains closed rank one geodesics if $H$ is a hemispherex.

\section{Existence of closed rank one geodesics}

In this section we discuss the existence of closed rank one geodesics in a finite FCC. Throughout this section $X$ denotes a finite FCC of dimension $n$, except in Section 3.4.

\subsection{Vertex links and 1-skeleton}

Recall that $X$ is dimensionally homogeneous, geodesically complete and has nonpositive curvature. It follows that for each vertex $v$ of $X$, the link $\operatorname{Link}(X, v)$ is dimensionally homogeneous, has no boundary and is a flag complex.

Recall that the set of edges in $X$ is a disjoint union: $E=\amalg_{i=1}^{n} E_{i}$. Let $v$ be a vertex of $X$. For $1 \leq i \leq n$, let $V_{i, v}$ be the set of vertices in $\operatorname{Link}(X, v)$ that correspond to edges in $E_{i}$. Since $X$ has no boundary, $V_{i, v}$ contains at least two points.

Lemma 3.1 Let $v \in X$ be a vertex and $1 \leq i \leq n$. If $V_{i, v}$ consists of exactly two points, then $\operatorname{Link}(X, v)$ is the spherical join of $V_{i, v}$ and $L_{i, v}$, where $L_{i, v}$ is the subcomplex of $\operatorname{Link}(X, v)$ consisting of all simplices of $\operatorname{Link}(X, v)$ with vertices in $V_{j, v}, j \neq i$.

Proof Denote by $\xi_{+}, \xi_{-}$the two vertices in $V_{i, v}$, and let $\eta \in V_{j, v}$ with $j \neq i$. Since $\operatorname{Link}(X, v)$ is a flag complex it suffices to show that there are edges $\eta \xi_{+}$ and $\eta \xi_{-}$in $\operatorname{Link}(X, v)$. 
Since $\operatorname{Link}(X, v)$ is dimensionally homogeneous, there is a $(n-1)$-simplex $\Delta_{1}$ of $\operatorname{Link}(X, v)$ containing $\eta . \Delta_{1}$ contains exactly one vertex from $V_{i, v}$. Without loss of generality we may assume it is $\xi_{+}$(thus there is an edge $\eta \xi_{+}$). Let $\Delta_{1}^{\prime} \subset$ $\Delta_{1}$ be the $(n-2)$-face disjoint from $\xi_{+}$. Since $\operatorname{Link}(X, v)$ has no boundary, there is a $(n-1)$-simplex $\Delta_{2} \neq \Delta_{1}$ containing $\Delta_{1}^{\prime}$. The assumption that $V_{i, v}$ consists of exactly two points implies $\xi_{-}$is a vertex of $\Delta_{2}$. In particular there is an edge $\eta \xi_{-}$.

For any vertex $v$ of $X$ and $1 \leq i \leq n$, let $X_{i, v}$ be the component of $X_{\{i\}}$ that contains $v$.

Corollary 3.2 Let $v \in X$ be a vertex and $1 \leq i \leq n$. If there are $\xi \in V_{j, v}$, $\eta \in V_{i, v}$ with $j \neq i$ and $d_{v}(\xi, \eta) \geq \pi$, then $X_{i, v}$ is not a circle.

Proof By Lemma 3.1. $V_{i, v}$ contains at least three points and therefore there are at least three edges of $X_{i, v}$ incident to $v$.

For an oriented edge $e, \bar{e}$ denotes the same edge with the opposite orientation and $t(e)$ denotes the terminal point of $e$. It is not hard to prove the following lemma.

Lemma 3.3 Let $\Gamma$ be a connected finite graph such that the valence of each vertex is at least two. Assume $\Gamma$ is not homeomorphic to a circle. Then for any two oriented edges $e_{1}, e_{2}$ in $\Gamma$, there is a geodesic $c$ from $t\left(e_{1}\right)$ to $t\left(e_{2}\right)$ such that $\bar{e}_{2} * c * e_{1}$ is also a geodesic. In particular, for any oriented edge $e$ of $\Gamma$, there is a geodesic loop $c$ based at $t(e)$ such that $\bar{e} * c * e$ is also a geodesic.

\subsection{Closed rank one geodesics}

Let $v \in X$ be a vertex and $\xi \in \operatorname{Link}(X, v)$. Then $\xi$ lies in the interior of a simplex $\Delta$ of $\operatorname{Link}(X, v)$. Define $T(\xi)=\left\{i: V_{i, v} \cap \Delta \neq \phi\right\}$. Then $T(\xi) \subset$ $\{1,2, \cdots, n\}$. We say $T(\xi)$ is the type of $\xi$. For an oriented edge $e$ of $X$ with initial point $v$, define $P_{e}=\left\{\xi \in \operatorname{Link}(X, v): d_{v}(\xi, \vec{e})=\pi / 2\right\}$.

Lemma 3.4 Let $e$ be an oriented edge of $X$. Then there is an isometry $D_{e}: P_{e} \rightarrow P_{\bar{e}}$ such that $\xi$ and $D_{e}(\xi)$ have the same type for any $\xi \in P_{e}$. 
Proof Let $i$ be the index such that the geometric edge of $e$ lies in $E_{i}, m$ the midpoint of $e$ and $Y$ the component of $H_{i}$ containing $m$. Denote by $v$ and $w$ the initial and terminal points of $e$, and $B_{0}$ and $B_{1}$ the components of $X_{T_{i}}$ containing $v$ and $w$ respectively. Since $g_{e_{Y}, 0}: Y \rightarrow B_{0}$ and $g_{e_{Y}, 1}: Y \rightarrow B_{1}$ are local isometric embeddings and $B_{0}, B_{1}$ are locally convex in $X$, the induced $\operatorname{maps} h_{0}: \operatorname{Link}(Y, m) \rightarrow \operatorname{Link}\left(B_{0}, v\right) \subset \operatorname{Link}(X, v)$ and $h_{1}: \operatorname{Link}(Y, m) \rightarrow$ $\operatorname{Link}\left(B_{1}, w\right) \subset \operatorname{Link}(X, w)$ are isometric embeddings. It is not hard to check that the images of these maps are $P_{e}$ and $P_{\bar{e}}$ respectively. Set $D_{e}=h_{1} \circ h_{0}{ }^{-1}$. Then $D_{e}: P_{e} \rightarrow P_{\bar{e}}$ is an isometry. Since $h_{0}$ and $h_{1}$ clearly preserve type, $D_{e}$ also preserves type.

Corollary 3.5 Let $X$ be a FCC , $v \in X$ a vertex and $e_{1}, e_{2}, e_{3}$ three oriented edges with initial point $v$. Suppose $d_{v}\left(\overrightarrow{e_{1}}, \overrightarrow{e_{3}}\right)=d_{v}\left(\overrightarrow{e_{2}}, \overrightarrow{e_{3}}\right)=\pi / 2$. Then $d_{v}\left(\overrightarrow{e_{1}}, \overrightarrow{e_{2}}\right)=\pi$ if and only if $d_{w}\left(D_{e_{3}}\left(\overrightarrow{e_{1}}\right), D_{e_{3}}\left(\overrightarrow{e_{2}}\right)\right)=\pi$, where $w$ is the terminal point of $e_{3}$.

Proof It follows easily from the fact that $D_{e_{3}}: P_{e_{3}} \rightarrow P_{\bar{e}_{3}}$ is an isometry.

We say a geodesic in a metric space with nonpositive curvature has rank one if its lifts in the universal cover have rank one.

Proposition 3.6 Let $c \subset X$ be a closed geodesic that is contained in the 1skeleton. If for each $i, c$ contains at least one edge from $E_{i}$, then $c$ is a closed rank one geodesic.

Proof Let $\pi: \tilde{X} \rightarrow X$ be the universal cover of $X, \tilde{c}$ a lift of $c$ to $\tilde{X}$ and $\tilde{E}_{i}=\pi^{-1}\left(E_{i}\right)$ for $1 \leq i \leq n$. Note $\tilde{X}$ is also a FCC and the set $\tilde{E}$ of edges of $\tilde{X}$ has the decomposition into different colors $\tilde{E}=\amalg_{i=1}^{n} \tilde{E}_{i}$.

Assume the proposition is false. Then there is half flatplane $H$ bounding $\tilde{c}$, that is, $H=\operatorname{image}(f)$ where $f$ is an isometric embedding $f: \mathbb{R} \times[0, \infty) \rightarrow \tilde{X}$ with $f(\mathbb{R} \times\{0\})=\tilde{c}$. For any vertex $v \in \tilde{c}, H$ determines a unique point $\xi_{v} \in \operatorname{Link}(\tilde{X}, v)$ with the following property: for any oriented edge $e \subset \tilde{c}$ with initial point $v$ and terminal point $w, \xi_{v} \in P_{e}$ and $D_{e}\left(\xi_{v}\right)=\xi_{w}$. By Lemma 3.4 all $\xi_{v}$ have the same type $T \subset\{1,2, \cdots, n\}$. Let $i \in T$. By assumption there is an oriented edge $e \subset \tilde{c}$ with $e \in \tilde{E}_{i}$. Let $v$ be the initial point of $e$. Since $\operatorname{Link}(\tilde{X}, v)$ is an all right spherical complex and $\vec{e} \in \operatorname{Link}(\tilde{X}, v)$ is a vertex, $\xi_{v} \in P_{e}$ implies $d_{v}(\vec{e}, \xi)=\pi / 2$ for any vertex $\xi$ of the smallest simplex of $\operatorname{Link}(\tilde{X}, v)$ containing $\xi_{v}$. Since $T\left(\xi_{v}\right)=T \ni i$, the definition of type implies there is an oriented edge $e_{i} \in \tilde{E}_{i}$ with $d_{v}\left(\vec{e}, \overrightarrow{e_{i}}\right)=\pi / 2$. This contradicts to the facts that $e \in \tilde{E}_{i}$ and $\tilde{X}_{i, v}$ is locally convex in $\tilde{X}$. 
Let $v \in X$ be a vertex. We define a relation $\sim_{v}$ on the set $\{1,2, \cdots, n\}$ as follows: $i \sim_{v} j$ if and only if there are oriented edges $e_{1} \in E_{i}, e_{2} \in E_{j}$ with initial point $v$ such that $d_{v}\left(\overrightarrow{e_{1}}, \overrightarrow{e_{2}}\right) \geq \pi$.

Proposition 3.7 Let $X$ be a finite FCC of dimension $n$. For a fixed vertex $v \in$ $X$, if $T \subset\{1,2, \cdots, n\}$ is an equivalence class with respect to the equivalence relation generated by $\sim_{v}$, then there is a closed geodesic in the 1-skeleton of $X$ that contains at least one edge from $E_{i}$ for each $i \in T$.

Proof By the definition of the equivalence relation, we may assume $T=$ $\left\{i_{1}, i_{2}, \cdots, i_{m}\right\}$ such that for each $t, 2 \leq t \leq m$, there is some $j, 1 \leq j<t$ with $i_{t} \sim_{v} i_{j}$. Now we prove the following claim by induction on $k, 1 \leq k \leq m$ : there is a closed geodesic $c_{k}$ in the 1-skeleton of $X$ such that for each $j \leq k$, $c_{k}$ contains an edge belonging to $E_{i_{j}}$ and incident to $v$.

The claim is clear for $k=1$. Now let $k \geq 2$ and assume the claim has been established for $k-1$. By the above paragraph we have $i_{k} \sim_{v} i_{j}$ for some $j<k$. Hence there are oriented edges $e_{j}^{\prime} \in E_{i_{j}}, e_{k} \in E_{i_{k}}$ with initial point $v$ such that $d_{v}\left(\overrightarrow{e_{j}^{\prime}}, \overrightarrow{e_{k}}\right) \geq \pi$. Corollary 3.2 implies $X_{i_{j}, v}$ and $X_{i_{k}, v}$ are not circles. By induction hypothesis, there is a closed geodesic $c_{k-1}$ in the 1-skeleton of $X$ containing an oriented edge $e_{j} \in E_{i_{j}}$ with initial point $v$. Let $v_{1}, v_{2}, v_{3}$ be the terminal points of the edges $e_{k}, e_{j}^{\prime}, e_{j}$ respectively. By Lemma 3.3 there is a geodesic loop $\tilde{c}_{1}$ in $X_{i_{k}, v}$ based at $v_{1}$ such that $c_{1}^{\prime}=\bar{e}_{k} * \tilde{c}_{1} * e_{k}$ is also a geodesic in $X_{i_{k}, v}$. Similarly there are geodesic loops $\tilde{c}_{2}$ and $\tilde{c}_{3}$ in $X_{i_{j}, v}$ based at $v_{2}$ and $v_{3}$ respectively such that $c_{2}^{\prime}=\bar{e}_{j}^{\prime} * \tilde{c}_{2} * e_{j}^{\prime}$ and $c_{3}^{\prime}=\bar{e}_{j} * \tilde{c}_{3} * e_{j}$ are geodesics in $X_{i_{j}, v}$. Proposition 2.3 implies $c_{1}^{\prime}, c_{2}^{\prime}$ and $c_{3}^{\prime}$ are geodesics in $X$. We reparametrize the closed geodesic $c_{k-1}$ so that it starts from $v$ with initial segment $e_{j}$. Define $c_{k}=c_{2}^{\prime} * c_{3}^{\prime} * c_{k-1} * c_{2}^{\prime} * c_{1}^{\prime}$ if $e_{j}^{\prime} \neq e_{j}$ and $c_{k}=c_{1}^{\prime} * c_{2}^{\prime} * c_{k-1}$ if $e_{j}^{\prime}=e_{j}$. Now it is easy to check that $c_{k}$ is a closed geodesic with the required property.

The following corollary follows immediately from Propositions [3.6 and 3.7

Corollary 3.8 Let $X$ be a finite FCC of dimension $n$ and $v \in X$ a vertex. If $\{1,2, \cdots, n\}$ is a single equivalence class with respect to the equivalence relation generated by $\sim_{v}$, then there is a closed rank one geodesic contained in the 1-skeleton of $X$.

Let $H$ be a hemispherex with central sphere $\mathbb{S}^{n}$, and $E_{1}, \cdots, E_{n+1}$ the equators of $\mathbb{S}^{n}$. For each $i, 1 \leq i \leq n+1$, let $H_{i}$ be a fixed hemisphere of $H$ that is 
attached to $\mathbb{S}^{n}$ along $E_{i}$. Denote the pole of $H_{i}$ by $p_{i}$. Then the distance between $p_{i}$ and $p_{j}$ is $\pi$ for $i \neq j$. Now let $X$ be a FCC and $v \in X$ a vertex with $\operatorname{Link}(X, v)=H$. Then there are oriented edges $e_{i}, 1 \leq i \leq n+1$ with initial point $v$ and $\overrightarrow{e_{i}}=p_{i}$. It is clear that $e_{i}$ and $e_{j}$ have different colors for $i \neq j$. It follows that the assumption in the above corollary is satisfied if the vertex link $\operatorname{Link}(X, v)$ is a hemispherex. Thus we have:

Corollary 3.9 Let $X$ be a finite FCC of dimension $n$. Suppose there is a vertex $v \in X$ such that $\operatorname{Link}(X, v)$ is a hemispherex, then $X$ has a closed rank one geodesic contained in the 1 -skeleton.

Proposition 3.10 Let $X$ be a finite FCC . Suppose there is a vertex $v \in X$ and $\xi \in V_{i, v}, \eta \in V_{j, v}$ with $i \neq j$ and $d_{v}(\xi, \eta)>\pi$, then $X$ contains a closed rank one geodesic in the 1-skeleton.

Proof Let $e_{1}$ and $e_{2}$ be the two oriented edges with initial point $v$ that give rise to $\xi$ and $\eta$ respectively. Since $d_{v}(\xi, \eta)>\pi$, Corollary 3.2 implies $X_{i, v}$ and $X_{j, v}$ are not circles. Lemma 3.3 then implies there are geodesic loops $c_{1} \subset X_{i, v}$ and $c_{2} \subset X_{j, v}$ based at $t\left(e_{1}\right)$ and $t\left(e_{2}\right)$ respectively such that $c_{1}^{\prime}:=\bar{e}_{1} * c_{1} * e_{1}$ and $c_{2}^{\prime}:=\bar{e}_{2} * c_{2} * e_{2}$ are geodesics in $X_{i, v}$ and $X_{j, v}$ respectively. Since by Proposition 2.3 $X_{i, v}$ and $X_{j, v}$ are locally convex in $X, c_{1}^{\prime}$ and $c_{2}^{\prime}$ are geodesics in $X$. Let $c=c_{2}^{\prime} * c_{1}^{\prime}$. Since $d_{v}(\xi, \eta)>\pi$, it is clear that $c$ is a closed rank one geodesic.

\subsection{A splitting criterion}

Let $Y$ be a $\mathrm{CAT}(0)$ space and $Z_{1}, Z_{2} \subset Y$ be two closed, convex subsets. We say $Z_{1}, Z_{2}$ are parallel if for some $a \geq 0$ there is an isometric embedding $f: Z_{1} \times[0, a] \rightarrow Y$ such that $f\left(Z_{1} \times\{0\}\right)=Z_{1}$ and $f\left(Z_{1} \times\{a\}\right)=Z_{2}$. For any closed convex subset $Z \subset Y$ of a $\operatorname{CAT}(0)$ space $Y$, let $P_{Z}$ be the union of all closed convex subsets that are parallel to $Z$. When $Z$ is geodesically complete, $P_{Z}$ is closed, convex and isometrically splits $Z \times C$, where $C \subset Y$ is closed and convex $([\mathrm{BBr} 2$, p.6).

Proposition 3.11 Let $X$ be a FCC of dimension $n$. Suppose $\{1,2, \cdots, n\}$ is the disjoint union of nonempty subsets $T, S$ with the following property: for any vertex $v \in X$, and any two edges incident to $v, e_{i} \in E_{i}, e_{j} \in E_{j}$ with $i \in T, j \in S$, there is a square containing $e_{i}$ and $e_{j}$ in the boundary. Then the universal cover $\tilde{X}$ of $X$ is isometric to the product of two CAT(0) FCCs . 
Proof For any vertex $v \in \tilde{X}$, let $\tilde{X}_{T, v}$ be the component of $\tilde{X}_{T}$ that contains $v$. We claim for any edge $e$ of $\tilde{X}$ with endpoints $v$ and $w, \tilde{X}_{T, v}$ and $\tilde{X}_{T, w}$ are parallel.

We may assume $e \in E_{i}$ for some $i \in S$, otherwise $\tilde{X}_{T, v}=\tilde{X}_{T, w}$. For any $k \geq 0$ we inductively define a subcomplex $\tilde{X}_{T, v}(k)$ of $\tilde{X}_{T, v}: \tilde{X}_{T, v}(0)=\{v\}$, for $k \geq 1$, $\tilde{X}_{T, v}(k)$ is the union of $\tilde{X}_{T, v}(k-1)$ and all the cubes in $\tilde{X}_{T, v}$ that have nonempty intersection with $\tilde{X}_{T, v}(k-1)$. Similarly one can define $\tilde{X}_{T, w}(k)$. We also define subcomplexes $\tilde{X}_{T, e}(k)$ of $\tilde{X}: \tilde{X}_{T, e}(0)=e$, for $k \geq 1, \tilde{X}_{T, e}(k)$ is the union of $\tilde{X}_{T, e}(k-1)$ and all the cubes whose edges are in $E_{i} \cup\left(\cup_{j \in T} E_{j}\right)$ and whose intersections with $\tilde{X}_{T, e}(k-1)$ contain edges from $E_{i}$. Set $\tilde{X}_{T, e}=\cup_{k \geq 0} \tilde{X}_{T, e}(k)$.

Since $\tilde{X}$ is a $\operatorname{CAT}(0)$ cubical complex, the vertex links of $\tilde{X}$ are flag complexes. Our assumption then implies that for any $(m-1)(m \leq n)$ cube $C$ in $\tilde{X}_{T, v}(1)$ or $\tilde{X}_{T, w}(1)$, there is a unique $m$-cube in $\tilde{X}$ that contains both $e$ and $C$. It follows that $\tilde{X}_{T, e}(1)$ contains $\tilde{X}_{T, v}(1)$ and $\tilde{X}_{T, w}(1)$ and there is an isomorphism

$$
f_{e, 1}: \tilde{X}_{T, v}(1) \times[0,1] \rightarrow \tilde{X}_{T, e}(1)
$$

such that $f_{e, 1 \mid \tilde{X}_{T, v}(1) \times\{0\}}$ is the identity map and $f_{e, 1}\left(\tilde{X}_{T, v}(1) \times\{1\}\right)=\tilde{X}_{T, w}(1)$. Now $\tilde{X}_{T, v}(k)=\cup_{v^{\prime}} \tilde{X}_{T, v^{\prime}}(1)$ and $\tilde{X}_{T, e}(k)=\cup_{e^{\prime}} \tilde{X}_{T, e^{\prime}}(1)$, where $v^{\prime}$ varies over all vertices in $\tilde{X}_{T, v}(k-1)$ and $e^{\prime} \subset \tilde{X}_{T, e}(k-1)$ varies over all edges from $E_{i}$. Notice all the maps $f_{e^{\prime}, 1}$ are compatible for $e^{\prime} \subset \tilde{X}_{T, e}(k-1)$ from $E_{i}$. It follows that for each $k$ there is an isomorphism $f_{e, k}: \tilde{X}_{T, v}(k) \times[0,1] \rightarrow \tilde{X}_{T, e}(k)$ such that $f_{e, k \mid \tilde{X}_{T, v}(k) \times\{0\}}$ is the identity map, $f_{e, k}\left(\tilde{X}_{T, v}(k) \times\{1\}\right)=\tilde{X}_{T, w}(k)$ and $f_{e, k}$ agrees with $f_{e, k-1}$ when restricted to $\tilde{X}_{T, v}(k-1) \times[0,1]$. The union of all these isomorphisms $f_{e, k}$ defines an isomorphism $f_{e}: \tilde{X}_{T, v} \times[0,1] \rightarrow \tilde{X}_{T, e}$ such that $f_{e \mid \tilde{X}_{T, v} \times\{0\}}$ is the identity map onto $\tilde{X}_{T, v}$ and $f_{e}\left(\tilde{X}_{T, v} \times\{1\}\right)=\tilde{X}_{T, w}$. It follows that $\tilde{X}_{T, v}$ and $\tilde{X}_{T, w}$ are parallel.

Fix a vertex $v_{0} \in \tilde{X}$ and let $P_{T}$ be the parallel set of $\tilde{X}_{T, v_{0}}$. Note $\tilde{X}_{T, v_{0}}$ is closed, convex and geodesically complete. It follows that $P_{T}$ isometrically splits $P_{T}=\tilde{X}_{T, v_{0}} \times Y$ where $Y \subset \tilde{X}$ is a closed convex subset and for $y \in Y$, $\tilde{X}_{T, v_{0}} \times\{y\}$ is parallel to $\tilde{X}_{T, v_{0}}$. By the claim we have established, all vertices of $\tilde{X}$ lie in $P_{T}$. Since $\tilde{X}$ is the convex hull of all its vertices we see $\tilde{X}=P_{T}$ splits.

Proposition 3.11 has previously been established in dimension 2 (Theorem 1.10 on p.36 of [W] and Theorem 10.2 in [BW]).

Let $X$ be a FCC. By Proposition 2.6 $X$ has the structure of a graph of spaces, where all the vertex and edge spaces are FCCs and the maps from edge spaces 
to vertex spaces are combinatorial immersions. The following corollary follows from Lemma 2.7 and Proposition 3.11

Corollary 3.12 Let $X$ be a FCC with a graph of spaces decomposition as in Proposition 2.6. If all the maps from edge spaces to vertex spaces are covering maps, then the universal cover of $X$ is isometric to the product of a simplicial tree and a $(n-1)$-dimensional $\mathrm{CAT}(0) \mathrm{FCC}$.

\subsection{Rank rigidity in low dimensions}

In this section we discuss the rank rigidity problem for finite FCCs with dimension $\leq 3$. A 1-dimensional finite FCC $X$ is a finite graph and each of its vertices is incident to at least two edges; $X$ clearly contains closed geodesics and all the geodesics in $X$ have rank one. The claim in dimension 2 follows easily from Corollary 3.8 and Proposition 3.11

Theorem 3.13 Let $X$ be a finite FCC of dimension 3 with universal cover $\tilde{X}$.

(1) If $\tilde{X}$ has higher rank, then $\tilde{X}$ is isometric to the product of two $\operatorname{CAT}(0)$ FCCs .

(2) If $\tilde{X}$ has rank one, then there is a closed rank one geodesic in the 1-skeleton of $X$.

Proof Suppose that $\tilde{X}$ does not split as a product. Recall the decomposition of the set of edges into different colors: $E=E_{1} \amalg E_{2} \amalg E_{3}$. We shall call the edges in $E_{1}, E_{2}, E_{3}$ blue, green and red edges respectively. By Proposition 3.10 and Proposition 3.11 we may assume the following: for any two oriented edges $e_{1}, e_{2}$ with the same initial point $v$ but different colors, $d_{v}\left(\overrightarrow{e_{1}}, \overrightarrow{e_{2}}\right) \leq \pi$ holds; there exist two oriented edges $e_{1}, e_{2}$ with different colors (say blue and green respectively) and the same initial point $v$ such that $d_{v}\left(\overrightarrow{e_{1}}, \overrightarrow{e_{2}}\right)=\pi$.

Consider the graph of spaces decomposition of $X$ where the vertex spaces are components of $X_{\{1,2\}}$. Let $G_{3}$ be the base graph. By Corollary 3.12 at least one of the maps from the edge spaces to vertex spaces is not a covering map. Recall the base graph $G_{3}$ is connected. Let $k \geq 0$ be the smallest integer with the following property: there are two vertices $v_{B}, v_{B^{\prime}}$ of $G_{3}$ at distance $k$ apart such that

(1) $B$ contains an oriented blue edge $e_{1}$ and an oriented green edge $e_{2}$ with the same initial point $v \in X$ such that $d_{v}\left(\overrightarrow{e_{1}}, \overrightarrow{e_{2}}\right)=\pi$; 
(2) there is an edge $e_{Y} \subset G_{3}$ incident to $v_{B^{\prime}}$ such that the map from the edge space $Y$ to the vertex space $B^{\prime}$ is not a covering map.

We claim $k=0$. Assume $k \geq 1$. Let $B_{0}=B, B_{1}, \cdots, B_{k}=B^{\prime}$ be a sequence of components of $X_{\{1,2\}}$ such that $v_{B_{i}}$ and $v_{B_{i+1}}(0 \leq i \leq k-1)$ are adjacent vertices in $G_{3}$. Since $k \geq 1$, the map from the edge space of $v_{B} v_{B_{1}}$ to the vertex space $B$ is a covering map. Lemma 2.7 implies that there is a red edge $e$ with one endpoint $v$ and the other endpoint $w$ in $B_{1}$ such that $e$ is perpendicular to both $e_{1}$ and $e_{2}$. Corollary 3.5 implies that there exist an oriented blue edge $e_{3}$ and an oriented green edge $e_{4}$ with initial point $w$ such that $d_{w}\left(\overrightarrow{e_{3}}, \overrightarrow{e_{4}}\right)=\pi$. Note $e_{3}, e_{4} \subset B_{1}$ and the distance from $v_{B_{1}}$ to $v_{B^{\prime}}$ in $G_{3}$ is $k-1$, contradicting to the definition of $k$. Therefore $k=0$ and $B=B^{\prime}$. By Lemma 2.7 there is a vertex $v^{\prime} \in B$ and oriented edges $e_{r}$ (red), $e_{b} \subset B$ with initial point $v^{\prime}$ such that $d_{v^{\prime}}\left(\overrightarrow{e_{r}}, \overrightarrow{e_{b}}\right)=\pi$. We may assume $e_{b}$ is a blue edge.

By Corollary 3.8 we may assume the following: for any vertex $v \in B$, if there are oriented blue edge $e_{1}$ and red edge $e_{2}$ with initial point $v$ such that $d_{v}\left(\overrightarrow{e_{1}}, \overrightarrow{e_{2}}\right)=\pi$, then all green edges incident to $v$ are perpendicular to all blue and red edges incident to $v$.

Recall that $B$ is a finite FCC of dimension 2. We consider the graph of spaces decomposition of $B$ where the vertex spaces consist of blue edges. Let $G$ be the connected base graph. Lemma 2.7 and the condition (1) above imply that not all maps from edge spaces to vertex spaces are covering maps. Let $l \geq 0$ be the smallest integer with the following property: there are two vertices $v_{C}$ and $v_{C^{\prime}}$ of $G$ at distance $l$ apart such that

(1) there is a vertex $v^{\prime} \in C$, an oriented red edge $e_{r}$ and an oriented blue edge $e_{b}$ with initial point $v^{\prime}$ such that $d_{v^{\prime}}\left(\overrightarrow{e_{r}}, \overrightarrow{e_{b}}\right)=\pi$;

(2) there is an edge $e_{Y} \subset G$ incident to $v_{C^{\prime}}$ such that the map from the edge space $Y$ to the vertex space $C^{\prime}$ is not a covering map.

Now the preceding paragraph and a similar argument as above show that $l=0$ and $C=C^{\prime}$.

There is a vertex $v^{\prime \prime} \in C$, an oriented blue edge $e_{b^{\prime}}$ and an oriented green edge $e_{g}$ with initial point $v^{\prime \prime}$ such that $d_{v^{\prime \prime}}\left(\overrightarrow{e_{b^{\prime}}}, \overrightarrow{e_{g}}\right)=\pi$. Corollary 3.2 implies $C=X_{1, v^{\prime \prime}}$ is not a circle. By Lemma 3.3 there is a geodesic $c \subset C$ from $v^{\prime}$ to $v^{\prime \prime}$ which starts with $e_{b}$ and ends with $\bar{e}_{b^{\prime}}$. Similarly there are geodesic loops $c_{1}$ and $c_{2}$ based at $t\left(e_{r}\right)$ and $t\left(e_{g}\right)$ respectively such that $c_{1}^{\prime}=\bar{e}_{r} * c_{1} * e_{r}$ and $c_{2}^{\prime}=\bar{e}_{g} * c_{2} * e_{g}$ are also geodesics. Set $c^{\prime}=c_{1}^{\prime} * \bar{c} * c_{2}^{\prime} * c$. Then $c^{\prime}$ is a closed geodesic that contains blue, green and red edges. By Proposition 3.6 $c^{\prime}$ is a rank one geodesic. 
Theorem 3.13 (1) also follows from a theorem of Ballmann and Brin [BBr2]: they proved that if a 3-dimensional, geodesically complete and piecewise Euclidean polyhedra $Y$ is $\mathrm{CAT}(0)$, has higher rank and admits a cocompact and properly discontinuous group of isometries, then $Y$ either isometrically splits or is a thick Euclidean building of type $\tilde{A}_{3}$ or $\tilde{B}_{3}$. A FCC certainly can not be an Euclidean building of type $\tilde{A}_{3}$ or $\tilde{B}_{3}$. The main point of Theorem 3.13 is the existence of closed rank one geodesics in rank one finite FCCs. Our proof of Theorem 3.13 is independent of the proof in $\mathrm{BBr} 2$.

\section{Tits alternative for foldable cubical complexes}

In this section we give a short proof of the Tits alternative for the fundamental group of a finite FCC. Ballmann and Swiatkowski have a slightly more general result $\mathrm{BSw}$.

Theorem 4.1 Let $X$ be a finite FCC . Then any subgroup of $\pi_{1}(X)$ either contains a free group of rank two or is virtually free abelian.

Proof We induct on the dimension of $X$. If $\operatorname{dim} X=1$, then $X$ is a finite graph and the Theorem clearly holds. Let $n=\operatorname{dim} X$ and $H$ a subgroup of $\pi_{1}(X)$. By Proposition 2.6 $X$ admits a graph of spaces decomposition where all the vertex and edge spaces are $(n-1)$-dimensional finite FCCs. It follows that $\pi_{1}(X)$ admits a graph of groups decomposition and acts on the associated Bass-Serre tree $T$. As a subgroup of $\pi_{1}(X), H$ also acts on $T$. By [PV], $H$ contains a free group of rank two unless one of the following happens: $H$ fixes a point in $T, H$ stabilizes a complete geodesic in $T$, or $H$ fixes a point in $\partial_{\infty} T$. We need to consider these three exceptional cases.

First assume $H$ fixes a point in $T$. Then $H$ fixes a vertex of $T$ and is a subgroup of a conjugate of some vertex group of the graph of groups decomposition for $\pi_{1}(X)$. We have observed that such a vertex group is the fundamental group of a finite FCC with dimension $n-1$. By induction hypothesis the claim on $H$ holds.

Now assume $H$ stabilizes a complete geodesic $c$ in $T$. Since $T$ is a simplicial tree, by taking an index two subgroup we may assume $H$ acts on $c$ as translations and so there is an exact sequence $1 \rightarrow N \rightarrow H \rightarrow \mathbb{Z} \rightarrow 1$. Thus $N \subset H$ has a fixed point in $c \subset T$. By the previous paragraph $N$ contains a free group of rank two or is virtually free abelian. We may assume $N$ is virtually free abelian. It implies that $H$ is virtually solvable. The claim on $H$ follows since 
any virtually solvable subgroup of a group acting properly and cocompactly by isometries on a CAT(0) space is virtually free abelian ( $[\mathrm{BH}]$, p.249). The case when $H$ fixes a point in $\partial_{\infty} T$ can be handled similarly.

\section{References}

[B] W. Ballmann, Lectures on spaces of nonpositive curvature, volume $\mathbf{2 5}$ of DMV seminar. Birkhaüser, Basel, 1995. MathReview

[BBr] W. Ballmann, M. Brin, Orbihedra of nonpositive curvature, Inst. Hautes Études Sci. Publ. Math. No. 82 (1995) 169-209 (1996). MathReview

[BBr2] W. Ballmann, M. Brin, Rank rigidity of Euclidean polyhedra, Amer. J. Math. 122 (2000) 873-885. MathReview

[BBu] W. Ballmann, S. Buyalo, Periodic rank one geodesics in Hadamard spaces, preprint 2002.

[BSw] W. Ballmann, J. Swiatkowski, On Groups acting on Nonpositively Curved Cubical Complexes, Enseign. Math. 45 (1999) 51-81. MathReview

[Br] M. Bridson, Geodesics and curvature in metric simplicial complexes, Group theory from a geometrical viewpoint (E.Ghys, A. Haefliger \& A. Verjovsky, eds.) Proceedings ICTP, Trieste, World Scientific, 1991. MathReview

[BH] M. Bridson, A. Haefliger, Metric spaces of nonpositive curvature, Grundlehren 319, Springer-Verlag, Berlin (1999). MathReview

[BW] M. Bridson, D. Wise, $\mathcal{V} H$ complexes, towers and subgroups of $F \times F$, Math. Proc. Cambridge Philos. Soc. 126 (1999) 481-497. MathReview

[C] R. Charney, The Tits conjecture for locally reducible Artin groups, Internat. J. Algebra Comput. 10 (2000) 783-797. MathReview

[D] M. Davis, Nonpositive curvature and reflection groups, Handbook of geometric topology, 373-422, North-Holland, Amsterdam, 2002. MathReview

[DJS] M. Davis, T. Januszkiewicz, R. Scott, Nonpositive curvature of blow-ups, Selecta Math. 4 (1998) 491-547. MathReview

$[\mathrm{M}] \quad$ G. Moussong, Hyperbolic Coxeter groups, Ph.D. thesis, The Ohio State University, 1988.

[PV] I. Pays, A. Valette, Sous-groupes libres dans les groupes d'automorphismes d'arbres, Enseign. Math. 37 (1991) 151-174. MathReview

[SW] P. Scott, T. Wall, Topological methods in group theory, Homological group theory (Proc. Sympos. Durham, 1977) pp. 137-203, LMS Lecture Note Ser. 36, CUP, Cambridge-New York, 1979. MathReview

[W] D. Wise, Non-positively curved squared complexes, aperiodic tilings, and nonresidually finite groups, Ph.D. Thesis, Princeton University, 1996.

Dept. of Math. Sciences, University of Cincinnati, Cincinnati, OH 45221, USA

Email: xiexg@ucmail.uc.edu

Received: 19 September 2003 Revised: 14 May 2004 